\title{
La poétique de Haeckel
}

\section{Nicolas Wanlin}

\section{(2) OpenEdition}

Journals

Édition électronique

URL : http://journals.openedition.org/aes/1161

DOI : 10.4000/aes.1161

ISSN : 2258-093X

\section{Éditeur}

Laboratoire LISAA

\section{Référence électronique}

Nicolas Wanlin, «La poétique de Haeckel », Arts et Savoirs [En ligne], 9 | 2018, mis en ligne le 14 mai 2018, consulté le 30 avril 2019. URL : http://journals.openedition.org/aes/1161 ; DOI : 10.4000/ aes. 1161

Ce document a été généré automatiquement le 30 avril 2019

Centre de recherche LISAA (Littératures SAvoirs et Arts) 


\title{
La poétique de Haeckel
}

\author{
Nicolas Wanlin
}

\section{NOTE DE L'AUTEUR}

Cet article a été conçu dans le cadre du programme ANR-DFG « Biolographes » (dir. Gisèle Séginger et Thomas Klinkert). Il a paru, pour la première fois, en allemand, sous le titre „Die Poetik Haeckels“ dans Lendemains. Études comparées sur la France 41, 162/163, 2016, p. 83-99 (version en ligne : URL : http://periodicals.narr.de/index.php/Lendemains/article/ view/2938). 
1 Au sein du tout organique que constitue la culture, différents domaines entretiennent des relations de divers ordres: indifférence, domination, subordination, rivalité, modélisation ou imitation, etc. Ainsi, les lettres et les sciences voient, au $\mathrm{XIX}^{\mathrm{e}}$ siècle, leurs relations évoluer et poser des problèmes. Or Haeckel prend explicitement comme objet de ses réflexions un domaine des lettres et sa relation aux sciences naturelles. Il s'agit de cette part de la culture que l'on appelle poésie, ou littérature ou encore

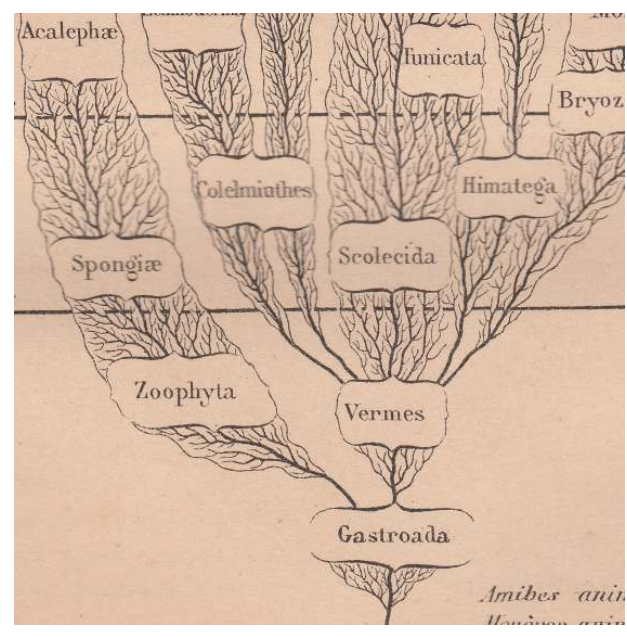
mythologie. Elle n'est pas en elle-même opposée à la science, ou dans une relation conflictuelle par sa nature même, mais le savant éprouve le besoin de différencier sa position et sa pratique scientifique par rapport à ce pôle culturel, pour mieux définir et réguler sa propre manière d'écrire, c'està-dire sa poétique.

\section{Haeckel et la poésie}

\section{La science contre les mythes}

2 Dans son Anthropogénie, Haeckel fait des observations sur la manière dont le grand naturaliste Linné s'est satisfait du mythe mosaïque de la création des espèces et du déluge et a même justifié le mythe de l'arche de Noé1. Haeckel est bien sûr critique à l'égard de ce trop grand respect pour les mythes bibliques. Pour lui, la science, et particulièrement la science évolutionniste, doit se défaire des manières de penser littéraires et mythologiques.

3 Ainsi, la première chose à faire est selon lui de débarrasser la science de l'anthropomorphisme, dans tout ce qui a trait à la création du monde et à la création de la vie, des espèces d'être vivants, qu'ils soient végétaux ou animaux, humains compris. Alors que le mode de pensée dominant est encore au $x_{x}{ }^{e}$ siècle (mais peut-être aussi aujourd'hui) religieux, façonnant l'image d'un dieu créateur à la ressemblance de l'homme, Haeckel assure que la théorie de l'évolution permet d'expliquer la création sans recourir à cet artifice anthropomorphique. Et ainsi,

L'obscur fantôme enfanté par la poésie mythologique s'évanouit devant l'éclatante lumière d'une connaissance scientifique des lois naturelles. ${ }^{2}$

En fait, c'est toute la connaissance du monde telle qu'elle est véhiculée par les poètes, ou les savants qui approuvent les poètes, qui est récusée par Haeckel. Il s'en prend ainsi, dans l'Histoire de la création naturelle au mythe de l'harmonie naturelle :

En quelque coin de la nature que vous portiez vos regards, vous ne rencontrerez pas cette paix idyllique chantée par les poètes; partout au contraire vous verrez la guerre, l'effort pour exterminer le plus proche voisin, l'antagoniste immédiat. Passion et égoïsme, voilà, que l'on en ait ou non conscience, le ressort de la vie. Le dicton poétique si connu :

"La nature est parfaite, partout où l'homme n'y introduit pas son tourment. »

Ce dicton ne manque pas de beauté ; mais il n'est malheureusement pas vrai. ${ }^{3}$ 
Il vise ainsi la conception providentialiste d'un monde harmonieux, qui avait cours chez les classiques comme chez la plupart des premiers romantiques. Dans le domaine français, les plus brillantes expressions de ce paradigme avaient été les Harmonies de la nature de Bernardin de Saint-Pierre et les Harmonies poétiques et religieuses de Lamartine ${ }^{4}$. Haeckel s'en prend donc aux conceptions poétiques en raison de leur anthropomorphisme, du mythe de l'harmonie naturelle, et pour une troisième raison: le téléologisme qui accompagne généralement les deux précédentes tares dans les conceptions poétiques. C'est pourquoi il accorde peu d'importance aux cosmogonies traditionnelles qui doivent être négligées, comme pré-scientifiques :

Il n'est nullement dans mon intention de vous entretenir de tant de cosmogonies poétiques, imaginées par les diverses espèces, races ou tribus humaines. Tout intéressant et fécond que soit un tel examen au point de vue ethnographique et à celui de l'histoire de la civilisation, il nous entraînerait beaucoup trop loin. En outre, la plupart de ces légendes cosmogoniques ont un caractère tellement fantaisiste, toute connaissance sérieuse de la nature y fait tellement défaut que, pour un examen scientifique de l'histoire de la création, elles manquent absolument d'intérêt. Je me bornerai donc à exposer une seule de toutes les cosmogonies imaginaires, la cosmogonie mosaïque, à cause de l'énorme influence que cette légende orientale a exercée sur la civilisation occidentale, puis je passerai aux hypothèses de ce genre ayant un caractère scientifique et qui ont été formulées pour la première fois par Linné, au commencement du siècle dernier. ${ }^{5}$

6 Cette posture rhétorique est étonnante. On pourrait s'attendre soit à une impasse pure et simple sur les cosmogonies mythologiques - ce que font la plupart des auteurs à cette époque - soit à une étude plus ou moins synthétique de la part de vérité qui se trouve dans toutes les cosmogonies. Mais Haeckel retient la Genèse biblique, qu'il appelle plaisamment une «légende orientale», parce qu'elle est l'occasion de surprendre ses détracteurs : il va relever dans la Genèse deux traits qu'il présentera comme cohérents avec la théorie de l'évolution : la division du travail et le perfectionnement ${ }^{6}$. Pour autant, même s'il se plaît à confirmer certaines idées de la Genèse, il en fait surtout la critique, notamment sur la question du Créateur :

Attribuer l'origine des premiers organismes terrestres, pères de tous les autres, à l'activité voulue et combinée d'un créateur personnel, c'est renoncer à en donner une explication scientifique, c'est quitter le terrain de la vraie science pour entrer dans le domaine de la croyance poétique (dichtenden Glaubenschaft), qui en est absolument distinct. ${ }^{7}$

7 En faisant ainsi la chasse à l'anthropomorphisme, sous toutes ses formes, Haeckel devait naturellement se heurter à un principe fondamental de l'esthétique classique, à savoir que dans les représentations littéraires, comme picturales, c'est l'humain qui doit être la mesure de tout, qui doit être le modèle et le sujet, explicite ou implicite, littéral ou allégorisé. C'est ce qui permet de comprendre que le discours haeckelien n'est finalement pas absolument anti-littéraire ou anti-artistique mais requiert une esthétique conforme à l'évolutionnisme, c'est-à-dire une esthétique qui se soit dépouillée de ses oripeaux anthropomorphiques. Il ne s'agit pas en fait de chasser complétement l'humain mais de le remettre à sa place, d'en faire le résultat d'une évolution et non une essence figée et extérieure aux réalités naturelles. 


\section{Pertinence et omniprésence de la poésie}

8 De fait, les livres de Haeckel sont truffés de poésie et d'art. Plus qu'aucun autre livre de science de la même époque, les livres de Haeckel citent fréquemment des poètes. Certes, le plus cité de ces poètes est Goethe, qui présente la singularité remarquable d'être à la fois une gloire poétique indiscutable mais également un naturaliste que Haeckel considère comme un des fondateurs de l'évolutionnisme.

Or, Haeckel ne trace pas de frontière nette et infranchissable entre les travaux de naturaliste de Goethe et son expression poétique. Dans l'Histoire de la création naturelle, Haeckel donne une épigraphe de Goethe en tête de chaque chapitre, du moins dans l'édition allemande. Le livre s'ouvre même sur la citation d'une grande page poétique de Goethe ${ }^{8}$. Mais Haeckel se permet de faire des coupes, sans le signaler, et de réduire ainsi une longue page du poète à trois petits paragraphes. Il opère donc déjà une transformation sur le texte poétique, ce qui montre qu'il se positionne lui-même comme un écrivain attentif à la composition et habile dans le choix des mots.

Dans la Generelle Morphologie, il cite aussi quatre vers d'une réplique de Méphistophélès dans Faust :

Qui veut reconnaître et détruire un être vivant commence par en chasser l'âme : alors il en a entre les mains toutes les parties; mais, hélas ! que manque-t-il ? rien que le lien intellectuel. 9

11 Par cette épigraphe, il se moque de l'analyse scientifique qui tue ce qu'elle veut définir et ne peut parler que d'êtres morts. La citation poétique est ainsi l'occasion d'un retour réflexif sur la pratique scientifique. Ce n'est plus seulement la science qui critique les idées poétiques mais la poésie qui, quand elle vient sous la plume d'un savant, peut toiser la science.

Dans l'Anthropogénie, il cite le Prométhée de Goethe puis à nouveau Faust, seize vers se concluant ainsi : "Le cercle de la terre m'est suffisamment connu. La vue sur l'autre monde nous est fermée. $»^{10}$

13 Haeckel utilise donc le paratexte non seulement pour invoquer une autorité tutélaire en la personne de Goethe mais il en fait aussi une transition pour passer de la méditation poétique et philosophique à l'histoire naturelle. Goethe est en effet une figure d'une double utilité : s'inscrire dans sa continuité confère une caution intellectuelle et autorise l'ouverture de la science naturelle sur la philosophie, voire sur la poésie. Je remarque toutefois que cette transition se fait peut-être plus facilement en Allemagne qu'en France en raison de traditions de pensée différentes. De fait, les citations de Goethe, particulièrement celles empruntées à son œuvre poétique, sont rarement traduites dans les éditions françaises. Le plus souvent, elles sont simplement supprimées.

Dans l'Histoire de la création naturelle, Haeckel cite encore des vers du poème didactique de Goethe sur La Métamorphose des animaux ${ }^{11}$. Goethe avait en effet écrit ces deux poèmes didactico-scientifiques, La Métamorphose des animaux et La Métamorphose des plantes, après avoir rédigé un essai en prose sur le même thème. Et Haeckel de faire remarquer que, parfois, chez Goethe, une excellente remarque de naturaliste est suivie d'une remarque fantaisiste et sans intérêt. Mais ce partage ne correspond pas au partage entre science et poésie ou science et philosophie. 
D'après lui, la littérature est susceptible d'une telle pertinence qu'il cite en exemple le roman de Goethe, Les Affinités électives ${ }^{12}$. L'analogie des affinités électives, c'est-à-dire le transfert d'un principe chimique au niveau des relations humaines, lui semble exemplaire de la continuité entre les disciplines. Il note que la différence entre affinités chimiques et affinités humaines tient au degré de complexité: les affinités humaines sont imprévisibles seulement parce qu'elles dépendent d'un trop grand nombre de facteurs ${ }^{13}$. Il n'en demeure pas moins qu'il y a une continuité épistémologique entre les sciences naturelles d'une part, la psychologie et la sociologie d'autre part. Par conséquent, le roman peut illustrer un aspect de la pensée scientifique contemporaine. De même, Haeckel est capable de reconnaître une sorte de pertinence globale de la poésie, un pouvoir de témoignage : toute la poésie et les arts sont, d'après lui, la conséquence de la reproduction sexuée ${ }^{14}$. Ainsi explique-t-il dans l'Anthropogénie, en se fondant sur Homère et la Bible, que l'importance de l'amour dans notre culture vient de la différenciation sexuelle et de la reproduction sexuée :

Or, tout cela a pour raison d'être l'union de deux cellules; et cet admirable phénomène provoque partout les effets les plus variés. Point d'acte organique qui puisse rivaliser avec celui-ci, même de loin, en puissance et en force de différenciation. En effet, le mythe sémitique d'Ève, qui séduisit Adam, pour l'amour du savoir, la vieille légende grecque de Pâris et d'Hélène, tant d'autres poèmes magnifiques n'expriment-ils pas simplement et poétiquement l'énorme influence que l'amour et la sélection sexuelle, qui en dépend, ont exercée dans l'histoire du monde, depuis la séparation des sexes ? L'influence de toutes les autres passions qui agitent le cœur humain ne saurait entrer en balance avec celle de l'amour, qui enflamme les sens et fascine la raison. D'un côté, nous célébrons dans l'amour la source des œuvres d'art les plus sublimes, des créations poétiques les plus nobles, de la musique, nous le vénérons comme le plus puissant facteur de la civilisation humaine, la cause première de la vie de famille et par suite de la vie sociale. D'autre part, nous redoutons l'amour comme une flamme destructive, c'est lui qui pousse le malheureux à sa perte c'est lui qui a enfanté plus de misère, plus de vice et de crime que toutes les calamités humaines ensemble. L'amour est si prodigieux, son influence est si énorme sur la vie psychique, sur les fonctions les plus dissemblables des centres nerveux, qu'on serait tenté, ici plus que partout ailleurs, de douter de l'effet surnaturel de notre explication naturelle. Néanmoins, la biologie comparée et l'histoire du développement nous conduisent sûrement, indubitablement, à la source la plus ancienne et la plus simple de l'amour, à l'affinité élective [Wahlverwandtschaft] de deux cellules différentes: la cellule spermatique et la cellule ovulaire. ${ }^{15}$

En reprenant le mot de Goethe, Wahlverwandtschaft (affinité élective), qui fait l'interface entre discours savant et discours littéraire, Haeckel justifie le contact entre les deux domaines.

\section{La tentation de la poésie}

Sur la base de cette justification, et de la caution de l'illustre poète-naturaliste Goethe, Haeckel s'autorise parfois à flirter avec la poésie. Ainsi, dans l'Anthropogénie, alors qu'il vient de décrire la fécondation de l'ovule par le spermatozoïde, il ajoute ceci :

Quelle belle occasion pour un poète de peindre sous les couleurs les plus brillantes le merveilleux phénomène de la fécondation! Il nous décrirait la rivalité des spermatozoaires, qui, enivrés de désir, tournoient en cadence autour des cellules ovulaires comblées d'hommages, s'engagent, à l'envi, dans les fins canalicules poreux du chorion, puis pénètrent «avec la conscience de leur acte» dans le 
protoplasme du jaune, où, se sacrifiant eux-mêmes, ils se dissolvent dans un moi meilleur. Les partisans de la téléologie pourraient aussi s'extasier devant la sagesse du créateur, qui a pratiqué dans la membrane cellulaire de nombreux canalicules poreux, afin de livrer passage aux animalcules spermatiques. Mais le naturaliste critique voit les choses beaucoup plus froidement et, pour lui, ce phénomène poétique, "cette couronne de la vie » est simplement la conjugaison de ceux cellules. $^{16}$

Sur le mode de la virtualité, il décrit le poème possible, l'interprétation téléologique possible, quasiment comme une prétérition : «je ne suis pas poète, donc je ne dirai pas que... d'ailleurs je suis naturaliste et donc je dirai que... » Mais le modèle sous-jacent de Goethe semble tellement puissant que le naturaliste tend à assumer le discours poétique et à lui laisser une place. La question qui se pose en fait est peut-être de savoir ce qui distingue, au fond, ces deux discours. Or, on sait que Goethe revendiquait une différence dans l'usage que l'artiste et le savant font de l'imagination. On le voit par exemple lorsqu'il écrit à Herder à propos de son idée de « plante primitive » (Urpflanze) :

Je suis sur le point de pénétrer enfin le mystère de la naissance et de l'organisation des plantes... La plante primitive sera la chose la plus singulière du monde, et la nature elle-même me l'enviera. Avec ce modèle et sa clef, on inventera une infinité de plantes nouvelles qui, si elles n'existent pas, pourraient exister, et qui, loin d'être le reflet d'une imagination artistique et poétique, auront une existence intime vraie, nécessaire même, et cette loi créatrice pourra s'appliquer à tout ce qui a une vie quelconque. ${ }^{17}$

Mais pour Haeckel, cette distinction entre la démarche de l'artiste et celle du savant doit surtout aboutir à équilibrer l'usage de la raison et de l'imagination dans l'écriture.

\section{L'équilibre de la raison et de l'imagination}

C'est à l'occasion d'une comparaison entre Charles Darwin et son grand-père Erasmus que Haeckel fait une mise au point sur le rôle de l'imagination dans l'histoire naturelle :

Conformément aux lois de l'hérédité ou atavisme, Érasme Darwin légua à son petitfils certaines vibrations de ses cellules ganglionnaires cérébrales, qui ne s'étaient nullement manifestées chez son fils. Ce fait est d'un haut intérêt pour la théorie de l'atavisme, que Charles Darwin a discutée avec tant de supériorité. D'ailleurs chez Érasme Darwin l'imagination créatrice l'emportait de beaucoup sur la raison critique, tandis que ces facultés sont parfaitement équilibrées chez le petit-fils. De nos jours, nombre de naturalistes à courte vue prétendent qu'en biologie, l'imagination est une qualité superflue ; à leurs yeux, son absence est un avantage, une garantie "d'exactitude ". Il ne sera donc pas hors de propos de rappeler à ce sujet la judicieuse manière de voir d'un grand naturaliste, qui fut même un des chefs de l'école strictement empirique ou soi-disant exacte. Jean Muller, le Cuvier allemand, dont les travaux seront toujours des modèles d'exactitude, a déclaré que l'effort combiné, l'harmonieux équilibre de l'imagination et de la raison étaient les conditions indispensables des grandes découvertes. J'ai choisi ce passage pour épigraphe de ma dix-huitième leçon. ${ }^{18}$

21 Ainsi, chez le grand-père, le naturaliste était trop poète ; mais on arrive à l'équilibre chez le petit-fils. Entre-temps, le père de Charles, Robert Darwin avait été totalement épargné par ces « vibrations des cellules ganglionnaires cérébrales » ataviques, la transmission des caractères ayant "sauté » une génération. Haeckel a donc une position modérée et assez accueillante pour l'imagination dans les sciences naturelles, même l'imagination des poètes, pourvu qu'elle soit équilibrée par la raison. 


\section{Poétique et rhétorique de Haeckel}

22 C'est sur la base de ces positions prudentes mais bienveillantes à l'égard de l'imagination et de l'expression poétique que Haeckel élabore sa propre écriture scientifique. Elle lui vaut un grand succès auprès d'un lectorat amateur de sciences mais non spécialiste qui reconnaît ses qualités. Ainsi Flaubert écrit-il à Georges Sand : «Joli bouquin, joli bouquin ! Le darwinisme m'y semble plus clairement expliqué que dans les livres de Darwin, même ! ${ }^{19}$ Et même un farouche opposant tel que Paul Bourget doit reconnaître à Haeckel un certain talent rhétorique, ne serait-ce que pour créer le scandale : « Haeckel avait [...] le don des phrases scandalisantes. [...] Ces formules outrancières révèlent un goût puéril d'étonner qui ne convient guère à un savant. $»^{20}$ Qu'on la prenne en bonne ou en mauvaise part, l'écriture de Haeckel suscite les réactions et fait émerger les enjeux épistémologiques. Il en a d'ailleurs conscience et thématise la question en faisant la leçon à d'autres savants dans Les Merveilles de la vie:

On devrait également éviter d'appeler organismes des corps inorganiques tels que la mer ou le globe terrestre. Ces désignations qui reposent sur une comparaison symbolique doivent être réservées à la poésie. Ainsi le mouvement rythmique des vagues devient pour le poète la respiration de la mer, leurs mugissements sont sa voix. Certains philosophes naturels (par exemple Fechner) conçoivent toute la terre comme un organisme géant, dont les innombrables organes ont été reliés en un tout harmonieux par l'intelligence mondiale (ou Dieu). De même le physiologiste Preyer considère les astres comme des «organismes brûlants dont l'haleine est peut-être de la vapeur de fer, dont le sang est du métal fondu, et qui s'alimentent peut-être de météorites ». Les dangers de ces métaphores ressortent justement de cet exemple, car Preyer a construit sur elles une hypothèse tout à fait insoutenable de la création. ${ }^{21}$

Là encore, la mise en garde tient un peu de la prétérition dans la mesure où Haeckel rapporte les analogies fallacieuses qu'il dénonce. Ces phrases illustrent donc bien que Haeckel assume cette prérogative scientifique qui consiste, du moins au XIX ${ }^{\mathrm{e}}$ siècle, à définir les limites des usages métaphoriques dans le discours scientifique, à légitimer certains usages analogiques et à en interdire d'autres. C'est donc à cette aune que l'on peut tâcher d'apprécier dans quelle mesure Haeckel s'appliquait à lui-même ses recommandations.

\section{Rendre sensible la « récapitulation »}

C'est sur une de ses idées les plus importantes, la récapitulation, que l'on peut apprécier la poétique haeckelienne en action, notamment dans les trois livres majeurs qui sont cités ici, la Generelle Morphologie, l'Anthropogénie et l'Histoire de la création naturelle :

L'ontogénèse, ou l'évolution individuelle, est une courte et rapide récapitulation de la phylogénèse, ou du développement du groupe correspondant, c'est-à-dire de la chaîne ancestrale de l'individu, et cette ontogénèse s'effectue conformément aux lois de l'hérédité et de l'adaptation. ${ }^{22}$

Dans l'Histoire de la création naturelle, il parle aussi, un peu plus loin d'un " parallélisme si important entre les deux évolutions individuelle et collective $»^{23}$. Ce "parallélisme», cette articulation de deux ordres de faits, articule aussi deux disciplines scientifiques, l'embryologie et la phylogénie, c'est-à-dire l'histoire de l'évolution des espèces. Aussi l' Anthropogénie est-elle composée de cinq chapitres faisant le bilan de l'embryologie et de la 
phylogénie jusqu'à Darwin, des neuf chapitres suivants qui traitent de l'embryologie, principalement humaine, c'est-à-dire de l'ontogénie, et des cinq suivants qui traitent de la généalogie de l'homme, c'est-à-dire de sa phylogénie. Du point de vue de la composition, la structure essentielle de l'ouvrage est une articulation entre l'ontogénie et la phylogénie. De fait, tout l'enjeu du livre, et non seulement des cinq premières leçons, est de montrer l'intérêt de lier dans une même étude l'ontogénie et la phylogénie. Et cette observation se confirme au niveau de la rhétorique de l'ouvrage, dans l'usage de la comparaison et du parallèle pour articuler les deux grands moments du livre.

En effet, selon Haeckel, la phylogénie seule nous dévoile les vraies causes de l'ontogénie : autrement dit, il faut commencer par établir un rapport, peut-être un parallèle entre les deux séries de faits pour ensuite voir dans l'une la cause de l'autre. C'est ce qu'on appellera la loi de récapitulation. Certes, l'idée de parallélisme entre ontogénie et phylogénie n'est pas nouvelle: elle date de la fin du xviII siècle. En revanche, ce qu'invente Haeckel est le discours qui transforme un simple parallélisme en un principe de récapitulation. Il commence à en formuler l'hypothèse dans la Generelle Morphologie, où il met en place les éléments de sa théorie évolutionniste, mais c'est dans l'Anthropogénie qu'il donne à son idée de récapitulation le statut d'une loi, énonçant que l'évolution de l'espèce est la "cause» du développement du germe, du fait de l'hérédité et de l'adaptation. Le statut de cette «loi » restera toujours problématique: Haeckel aurait voulu la faire reconnaître comme un principe fondamental par la communauté scientifique mais elle est refoulée à l'occasion d'une importante controverse scientifique ${ }^{24}$

Reprenant une méthode de l'anatomie comparée déjà utilisée par Étienne Geoffroy SaintHilaire, Haeckel tâche de mettre en évidence des ressemblances entre les embryons de différentes espèces animales, en les dessinant côte à côte et en énumérant les traits communs. Il escompte ainsi produire un effet d'évidence (evidentia) qui vaut pour une preuve. On lit par exemple ceci dans l'Anthropogénie:

De quelle valeur inestimable sont l'amphioxus et l'ascidie pour l'intelligence du développement humain et de la vraie nature de l'homme, vous avez pu vous en rendre compte en parcourant les tableaux des principales homologies entre les premiers et les derniers vertébrés. (Cinquième tableau.) Là vous avez trouvé la preuve irréfutable qu'au début de son développement, l'embryon humain ressemble, par les principaux traits de son organisation, à l'amphioxus et à l'ascidie, autant qu'il s'écarte de l'homme adulte. ${ }^{25}$

L'emploi du verbe ressembler (übereinstimmen) et du substantif homologie (Homologie) manifeste que Haeckel vise à faire percevoir des ressemblances là où on n'en voyait pas. Le bouleversement imaginaire qu'il souhaite consiste à faire admettre l'identité de ce qui est encore perçu comme irrémédiablement distinct, en remontant les étapes du processus de différenciation ${ }^{26}$.

Parmi ces étapes du développement, il faut noter, comme une des principales, la larve intestinale, la gastrula, dont nous venons de nous occuper, cette curieuse forme embryonnaire existant déjà chez les éponges, et qui se retrouve, identiquement la même, dans les classes zoologiques les plus dissemblables jusqu'aux vertébrés. ${ }^{27}$

Parmi les homologies rendues patentes, ou prétendues telles, la plus fondamentale est celle qui met en évidence la structure essentielle aux diverses formes animales, la gastrula . C'est tout simplement la formation d'un sac, ou une poche, qui est la forme de base que peut prendre un amas de cellules même très rudimentaire; or, pour Haeckel, ce sac est le 
prototype de toutes les formes de tube digestif existant chez les animaux, plus ou moins longs, plus ou moins complexes, ayant un seul ou plusieurs orifices. En ramenant une diversité de formes à une seule structure simple, tous les gasteres divers à une gastrula unique, Haeckel apparente c'est-à-dire crée une parenté entre toutes les formes animales.

Plus fondamentalement encore, l'opération rhétorique centrale de l'Anthropogénie, préparée par les livres précédents, consiste à rendre sensible et convaincante l'analogie entre l'ontogénèse et la phylogénèse c'est-à-dire la formation d'un individu pendant le temps de la gestation et l'évolution d'une espèce depuis l'apparition de la vie. La plus grande difficulté semble tenir à l'immense disproportion de durée des deux processus. Mais Haeckel tâche de faire de cette disproportion un argument :

Parlons d'abord du laps de temps qu'a mis le genre humain à se dégager du règne animal. La première pensée qui nous frappe est celle de l'énorme différence existant entre la durée de l'embryologie et celle de la phylogénie humaine. Le court espace de temps nécessaire à l'ontogenèse de l'individu humain s'évanouit devant l'infinie durée que réclame la phylogénèse du genre humain. ${ }^{28}$

Tout d'abord, l'attention est focalisée sur l'étonnant écart entre deux histoires, celle de l'embryon et celle de l'espèce (Keimes-Geschichte et Stammes-Geschichte), entre deux genèses (Onto-Genesis et Phylo-Genesis). Haeckel occulte ainsi au passage (probablement de bonne foi) le fait que la différence de durée recouvre en fait une différence de nature. Employer le même mot, Geschichte ou Genesis, pour l'individu et l'espèce est déjà un biais de raisonnement étonnant car ces deux objets n'ont pas du tout le même statut ontologique et ne sauraient donc être rapprochés par une analogie, encore moins par une homologie. Le parallélisme est donc arbitraire et construit par les mots. Mais le talent rhétorique de Haeckel consiste à focaliser l'attention sur une autre difficulté qu'il sait pouvoir vaincre :

Toujours la durée de l'ontogenèse est insignifiante, si on la compare à l'immense espace de temps qu'a nécessité la phylogénèse, l'évolution graduelle de la série des ancêtres. Cet espace de temps ne se mesure pas par années ou centaines d'années, mais par milliers et millions d'années. Il a fallu, en effet, bien des millions d'années pour que de l'antique ancêtre monocellulaire sortît graduellement le plus parfait des vertébrés, l'homme. En niant que l'homme descende des animaux inférieurs et, originellement, d'un ancêtre monocellulaire, en traitant ces faits de prodige incroyable, les adversaires de la théorie de la descendance oublient que le même prodige s'accomplit, pour chaque homme, dans le court espace de neuf mois. Cette série de formes, que nos ancêtres animaux ont mis des millions d'années à parcourir, chacun de nous la reproduit durant les quarante semaines de son existence dans le sein maternel.

Mais toutes ces métamorphoses organiques sont d'autant plus étonnantes, qu'elles s'effectuent plus rapidement. Par conséquent, si nos adversaires tiennent la descendance animale du genre humain pour un fait incroyable, l'évolution de l'individu humain à partir de l'ovule doit leur sembler plus merveilleuse encore. Cette métamorphose ontogénétique, qui s'accomplit sous nos yeux, l'emporte en merveilleux sur la métamorphose onto[sic pour phylo]génétique, autant que sa durée est plus courte. En effet, dans le court espace de quarante semaines, l'embryon humain doit évoluer de la cellule simple à l'homme adulte, tandis que, pour exécuter la même évolution, les ancêtres de l'homme ont eu besoin de bien des millions d'années. ${ }^{29}$

Haeckel retient ici quelques points d'analogie entre l'individu et l'espèce, l'ontogénèse et la phylogénèse et consolide le sentiment de cette analogie par l'usage d'un même terme pour désigner les deux réalités rapprochées: Geschichte (histoire), Entwickelung (développement), ou même Metamorphosen (métamorphoses) ou encore Genesis (genèse). 
De cette manière, Haeckel peut alors renverser l'ordre de ce qui est crédible et de ce qui est incroyable: la grossesse/gestation - pourtant universellement connue et admise, étudiée depuis longtemps et qui est le prodige de la création d'un être complexe à partir de cellules uniques simples et minuscules - est plus incroyable que l'évolution des espèces depuis la monère.

Mais surtout, alors que l'attention est attirée par ce tour de passe-passe rhétorique, ce qui est occulté au passage est l'absolue différence conceptuelle entre Keimes et Stammes pourtant traités en parallèle, entre l'individu en germe dans l'embryon et l'espèce. L'individu et l'espèce n'ont pas du tout le même statut ontologique, mais comme tout un chacun est prêt à admettre qu'un individu " évolue », cela fait admettre que l'espèce le peut aussi.

Ainsi, la poétique analogique de Haeckel, conformément à son programme, ne suit pas les mêmes chemins que la poétique mythologique ou la poétique romantique. Elle se donne bien de nouveaux moyens assortis à un nouvel imaginaire. S'il s'agit de métamorphose, cette métamorphose ne doit pas tout à la poésie ovidienne mais tire plutôt ses motifs des recherches scientifiques, notamment embryologiques et paléontologiques. Toutefois, il faut bien remarquer qu'elle dévoie un principe épistémologique cher à son siècle : l'observation.

Haeckel cherche à rendre visible, à faire apparaitre. Pour cela, il excède les procédures de l'observation et, plutôt qu'à la preuve, il en appelle à l'évidence et à l'étonnement l'évidence, c'est-à-dire ce qui saute aux yeux et, ipso facto, court-circuite la raison. Or, cette poétique fut couronnée de succès par un large public. Et le succès (c'est-à-dire à la fois la réussite et la diffusion) de la poétique haeckelienne ne se réduit pas à la poétique des textes écrits par Haeckel: ses textes ou leur vulgarisation ont favorisé l'appropriation, par des écrivains, de certains traits poétiques.

\section{La poésie haeckelienne}

36 L'influence de Haeckel sur les romanciers, surtout sur Flaubert, a déjà été étudiée mais on connaît moins la poésie qui fit écho aux théories évolutionnistes et précisément à celle de $\mathrm{Haeckel}^{30}$. En fait, il faut moins chercher des relations d'influence entre textes-sources et textes-cibles que des échos, des résonnances: les poèmes ne transposent pas, ni ne recodent un savoir scientifique nouveau, émergeant ex nihilo; il s'agit plutôt d'un imaginaire de l'évolution, commun à la poésie et aux sciences, qui saisit des occasions de s'actualiser tantôt dans les textes savants tantôt dans les poèmes, les uns et les autres s'échangeant des motifs et les reformulant.

37 Ainsi, chez Jules de Strada, auteur d'une monumentale Épopée humaine, dont un volume est consacré à La Genèse universelle, les cosmogonies mythologiques sont invoquées comme des précédents des théories qui formulent une origine animale de l'humanité :

Pourquoi le Sphinx ? Pourquoi le Centaure, le Faune?

L'homme pris dans la bête effroyable ou bouffonne,

Engainé, ne sortant qu'à moitié des taureaux?

Les animaux servant à l'homme de fourreaux?

Quel antique lien cachent ces alliances?

N'est-ce pas un lointain souvenir des enfances, Des temps où l'homme encor plongeait dans l'animal,

En sortait, peu à peu se levait triomphal? 
Et le biblique Éden mit ainsi que l'Asie

Sur l'homme et l'animal la même poésie... ${ }^{31}$ d'une origine minérale de la vie et de l'identité entre matière organique et inorganique. Certes, Haeckel n'était ni le seul ni le premier à formuler de telles idées mais il contribua grandement, par l'efficacité de sa poétique et la diffusion de ses livres, à en fixer les motifs dans l'imaginaire :

Or, dans les profondeurs

De l'Océan, je vis de magiques splendeurs

Étinceler soudain. Créatrice sublime,

La Lumière était là, couvant le large abîme.

Quelle œuvre s'ébauchait en ses divins rayons?

Traçait-elle, sous l'eau stérile, les sillons

Où germerait la riche et féconde semence

Des moissons à venir? Quel incendie immense

À cet ardent foyer allait-il s'allumer?

Verrais-je le granit informe s'animer

Et le roc brut pousser de vivaces racines ?32
L'hypothèse de l'origine minérale de la vie est ici figurée par un tel raccourci qu'elle est presque grotesque, même si la tournure interrogative y ajoute une nuance de prudence. Les idées transformistes ont toujours été caricaturées, le plus souvent dans le but de les discréditer et on ne compte plus les portraits de Darwin en singe... Mais l'ambivalence de ces images est que leur outrance même favorise leur imprégnation dans l'imaginaire et leur diffusion. Le poète prend d'ailleurs soin de mêler le lexique spiritualiste du thème traditionnel des « merveilles de la nature » à un lexique scientifique non encore acclimaté en poésie :

Ô miracles d'amour, germes, spores, ovules,

Saintes communions de brûlantes cellules,

Ferments générateurs qui se cherchent entre eux

Et s'embrassent, gonflés de levains chaleureux ;

Atomes, vibrions, infusoires, monades,

Microbes infinis, croissantes myriades

Qui pullulent dans l'air, grouillent, flottent partout... ${ }^{33}$

Jean Richepin, qui emprunta parfois le ton d'une poésie didactique un peu modernisée depuis Delille, se risque même à formuler de manière assez explicite la théorie de la récapitulation, dans une ode enthousiaste à la mer, berceau de la vie :

C'est par atavisme encore

Que dans l'œuf, où s'édulcore

Le mucus, tous les tétards,

Tous, et même aussi le nôtre,

Revivent l'un après l'autre 
Leurs liquides avatars.

Dans le sein de notre mère, Chaque passage éphémère Où, fœtus, nous nous formons Représente un des passages Que connut aux anciens âges Notre être dans les limons.

Ainsi tous, tant que nous sommes, Les bêtes comme les hommes Nous rendons à notre insu Inconscient témoignage Aux sources de ce lignage Qui de la mer est issu. ${ }^{34}$

Par le choix du terme "limons", Richepin tisse un lien entre le récit biblique de la création d'Adam et la " gelée primordiale ", le bathybius dans lequel Haeckel voulait voir la forme la plus primitive de la vie ; par le terme d' " avatars », il laisse imaginer, dans les étapes de l'embryogénèse, une interprétation scientifique de l'idée bouddhiste de réincarnation. Car c'est par syncrétisme que cristallisent les images poétiques: le transformisme, le bouddhisme, mais aussi la philosophie de Schopenhauer, parfois celle de Platon, et bien d'autres ingrédients encore incluant les traditions littéraires et iconographiques, participent à cette synthèse imaginaire. Henri Cazalis, le médecin-poète ami de Mallarmé, en donne un très bel exemple, aux consonances baudelairiennes, dans un poème qu'il dédie à Darwin et intitule « Réminiscences »:

Je sens un monde en moi de confuses pensées, Je sens obscurément que j'ai vécu toujours, Que j'ai longtemps erré dans les forêts passées, Et que la bête encor garde en moi ses amours. $[\ldots]$

Certains soirs, en errant dans les forêts natales, Je ressens dans ma chair les frissons d'autrefois, Quand, la nuit grandissant les formes végétales, Sauvage, halluciné, je rampais dans les bois.

Dans le sol primitif nos racines sont prises;

Notre âme, comme un arbre, a monté lentement ; Ma pensée est un temple aux antiques assises, Où l'ombre des Dieux morts vient errer par moment.

Quand mon esprit aspire à la pleine lumière, Je sens tout un passé qui me tient enchaîné ; Je sens rouler en moi l'obscurité première :

La terre était si sombre aux temps où je suis né !

Mon âme a trop dormi dans la nuit maternelle ; Pour atteindre le jour, qu'il m'a fallu d'efforts ! Je voudrais être pur : la honte originelle, Le vieux sang de la bête est resté dans mon corps..$^{35}$

Parmi bien des images communes aux différentes conceptions de l'évolution, les termes de «naissance » et de " nuit maternelle » évoquent ici une gestation de l'espèce analogue à la gestation de l'individu, donc une image de la récapitulation. Mais là encore, on ne 
peut concevoir la poésie comme une simple adaptation ou traduction de la science. Il s'agit bien plutôt d'un improbable syncrétisme entre plusieurs discours, plusieurs disciplines et domaines de la culture, où "La vie antérieure » de Baudelaire ne compte sans doute pas moins que les textes de Darwin et de Haeckel ${ }^{36}$. Ce qu'apporte le moment haeckelien dans l'histoire de l'imagination est donc moins une thèse scientifique à vulgariser que la reformulation et la remotivation de motifs traditionnels ainsi que la provocation à penser à nouveau et à diffuser l'idée de l'évolution de l'être, de son instabilité et de sa variation.

Ainsi, on peut parler de poétique haeckelienne dans la mesure où non seulement Haeckel a mis au point un discours efficace (avec son lexique, ses figures, ses arguments, ses motifs) et produit des représentations, mais aussi parce que cette production a fécondé les pratiques de représentation artistique et en particulier la poésie. La poétique haeckelienne est une manifestation de la solidarité et de l'interdépendance des discours, des disciplines et des domaines de la culture. Arts et sciences apparaissent bien comme les facettes différentes d'un même processus d'invention culturelle.

\section{NOTES}

1. Anthropogénie, ou Histoire de l'évolution humaine : leçons familières sur les principes de l'embryologie et de la phylogénie humaines [Anthropogenie oder Entwickelungsgeschichte des Menschen, 1874], trad. Ch. Letourneau, C. Reinwald, 1877, p. 50.

2. Histoire de la création des êtres organisés d'après les lois naturelles [abrégé par la suite, comme c'est l'usage, en Histoire de la création naturelle], trad. Ch. Letourneau, Paris, C. Reinwald, [1874] 1877, p. 11. L'édition française citée se fonde sur l'édition allemande de Natürliche Schöpfungs-Geschichte, Berlin, Druck und Verlag von Georg Reimer, 1868.

3. Ibid., p. 19. Il cite un vers célèbre de Schiller : „Die Natur ist vollkommen überall, / Wo der Mensch nicht hinkömmt mit seiner Qual.“ (Die Braut von Messina oder die feindlichen Brüder, 1803.)

4. Henri Bernardin de Saint-Pierre, Harmonies de la nature, Paris, Méquignon-Marvis, 1815 et Alphonse de Lamartine, Harmonies poétiques et religieuses, Paris, C. Gosselin, 1830.

5. Histoire de la création naturelle, op. cit., p. 31.

6. Ibid., p. 35-36.

7. Ibid., p. 281.

8. Goethe [Christoph Tobler], „Die Natur“, cité dans les éditions allemandes de Natürliche Schöpfungs-Geschichte. En fait, ce texte est de Christoph Tobler mais a longtemps été attribué à Goethe depuis sa première publication en 1783 dans le Tierfurter Journal (J.W.v. Goethe, Sämtliche Werke, Band 2.2: Erstes Weimarer Jahrzehnt 1775-1786, 2, München, Carl Hanser Verlag, 1987, p. 477-479).

9. Je cite ici la traduction de Nerval : Faust de Goethe suivi du Second Faust... traduits par Gérard [de Nerval], Librairie de Charles Gosselin, 1843, p. 55.

10. Ibid., p. 251.

11. Ibid., p. 79. 
12. Voir Ernst Haeckel, Essais de psychologie cellulaire [Die Perigenesis der Plastidule..., 1876], trad. Jules Soury, Paris, Librairie Germer Baillière, 1880, p. 41. Les Affinités électives [Die Wahlverwandschaften, 1809] furent traduites et publiées en France dès 1810 (Paris, S.-C. L'Huillier). 13. Ibid.

14. On peut voir là un précurseur du « darwinisme littéraire » qui sévit outre-Atlantique, théorie littéraire et méthode critique qui prétend expliquer les romans par la théorie de l'évolution darwinienne et la psychologie évolutionniste. Ce regard naturaliste sur la littérature trouve plutôt ses racines dans le spencerisme d'outre-Manche que dans Haeckel mais il est intéressant de voir que Haeckel aussi ouvrait cette porte, même si elle a eu peu de fortune dans nos pays. Voir à ce sujet le numéro 33 de la revue Figura, notamment mon article: Nicolas Wanlin, «Darwinismes littéraires. L'ancien et le nouveau, leurs présupposés et leurs limites », Figura, n - 33 : «Les voies de l'évolution. De la pertinence du darwinisme en littérature » dirigé par JeanFrançois Chassay, William Messier et Daniel Grenier, Montréal, UQAM, mai 2013.

15. Anthropogénie, op. cit., p. 578.

16. Ibid., p. 117-118.

17. Goethe à Herder, Naples, le 22 mai 1787, dans Mémoires de Goethe. II. Voyage en Italie, Paris, Charpentier, 1833, p. 178-179.

18. Anthropogénie, op. cit., p. 64-65.

19. Gustave Flaubert, Correspondance, éd. Jean Bruneau, coll. "Bibliothèque de la Pléiade ", Gallimard, vol. IV, 1998, p. 824.

20. Paul Bourget, « Haeckel et le pangermanisme », L'Illustration, n 3990, 23 août 1919, p. 146.

21. Les Merveilles de la vie : études de philosophie biologique pour servir de complément aux «Énigmes de l'univers » [Die Lebenswunder, 1904], Paris, Librairie Reinwald/Schleicher frères, 1907, p. 34-35.

22. Histoire de la création naturelle, op. cit., p. 225. Cette phrase est reprise à peu près telle quelle dans la Generelle Morphologie [Generelle Morphologie der Organismen, 1866], l'Histoire de la création naturelle et dans l'Anthropogénie.

23. Ibid., p. 226.

24. Voir Britta Rupp-Eisenreich, « Ernst Heinrich Haeckel » dans Patrick Tort dir., Dictionnaire du darwinisme et de l'évolution, Paris, PUF, 1996, t. 2, p. 2072-2114 et particulièrement p. 2090-2114.

25. Anthropogénie, op. cit., p. 300.

26. Sur le rôle de l'illustration dans l'argumentation et la médiatisation, voir Nick Hopwood, Haeckel's Embryos. Images, Evolution, and Fraud, Chicago, Chicago University Press, 2015.

27. Anthropogénie, op. cit., p. 302.

28. Ibid., p. 302-303.

29. Ibid., p. 303. (Je mets en gras.)

30. Sur la présence de Haeckel dans le roman, voir Gisèle Séginger, «La réécriture de Cuvier : la création du monde entre savoir et féerie ", Revue Flaubert, $\mathrm{n}^{\circ} 13$ (dir. Stéphanie Dord-Crouslé), 2013 et Juliette Azoulai, « De la rage métaphysique au calme scientifique : religion et sciences naturelles chez Flaubert ", Flaubert. Revue critique et génétique, $\mathrm{n}^{\circ} 13:$ «Flaubert, les sciences de la nature et de la vie (dir. G. Séginger), 2015. Sur la poésie évolutionniste, voir notamment Lionel Stevenson, Darwin among the Poets, Russel and Russel, 1963, John Holmes, Darwin's Bards : British and American Poetry in the Age of Evolution, Edinburgh, Edinburgh University Press, 2013 et mes articles, Nicolas Wanlin, «La poétique évolutionniste, de Darwin et Haeckel à Sully Prudhomme et René Ghil », Romantisme, n 154 : «Le vivant », dir. Gisèle Séginger, 2011-4 et "La poésie darwinienne et anti-darwinienne de 1860 à 1939 : de nouvelles images de l'humanité ", L'Héritage de Charles Darwin dans les cultures européennes, dir. Georges Letissier et Michel Prum, coll. « Racisme et eugénisme », L'Harmattan, 2011. Sur la poésie scientifique en général au XIX ${ }^{e}$ siècle, voir Hugues Marchal dir., Muses et Ptérodactyles. La poésie de la science de Chénier à Rimbaud, Éditions du Seuil, 2013. 
31. Jules Strada, L'Épopée humaine. La genèse universelle, 1890 donner si possible la référence complète.

32. Jean-Étienne Chamard, L'Épopée des âges. Les Origines, poème, Paris, L. Rodstein, [écrit entre 1874 et 1879] 1947.

33. Ibidem.

34. Richepin, Jean, « La gloire de l'eau », La Mer, 1886.

35. Cazalis, Henri, « Réminiscences » (à Charles Darwin), L'Illusion, 1875-1893.

36. Charles Baudelaire, « La vie antérieure », Les Fleurs du mal, 1857.

\section{RÉSUMÉS}

On examine ici tout d'abord le discours porté par le naturaliste Ernst Haeckel (1834-1919) sur la poésie et les mythes dans ses ouvrages scientifiques, oscillant entre rejet et reconnaissance. On observe ensuite comment Haeckel déploie sa propre rhétorique au service de son argumentation scientifique. Enfin, on relève des exemples de poèmes français qui font écho à Haeckel, constituant ce qu'on pourrait appeler une poésie haeckelienne.

This paper first examines the opinions of the naturalist Ernst Haeckel (1834-1919) about poetry and myths, parted between rejection and acknowledgment. Then, it presents the rhetorics of Haeckel himself when arguing his own scientific theories. Lastly, it points out French poems echoing Haeckel and forming what could be called a haeckelian poetry.

\section{INDEX}

Mots-clés : Haeckel (Ernst), poétique des sciences, évolutionnisme, récapitulation, poésie Keywords : Haeckel (Ernst), poetics of science, evolutionism, recapitulation, poetry

\section{AUTEUR}

\section{NICOLAS WANLIN}

École polytechnique (Paris-Saclay) / LinX / projet ANR « Biolographes » 